\title{
Investigation of ultra-thin waveguide arrays on a Bloch surface wave platform
}

\author{
Libo Yu, ${ }^{1}$ Elsie Barakat, ${ }^{1, *}$ Wataru Nakagawa, ${ }^{2}$ and Hans Peter Herzig ${ }^{1}$ \\ ${ }^{1}$ Ecole Polytechnique Fédérale de Lausanne, Optics \& Photonics Technology Laboratory, \\ Neuchâtel CH-2000, Switzerland \\ ${ }^{2}$ Montana State University, Department of Electrical and Computer Engineering, Bozeman, Montana 59717-3780, USA \\ ${ }^{*}$ Corresponding author: elsie.barakat@epfl.ch
}

Received August 19, 2014; revised October 15, 2014; accepted October 15, 2014; posted October 21, 2014 (Doc. ID 221228); published November 10, 2014

\begin{abstract}
Ultra-thin polymer optical waveguide couplers for integrated optics based on Bloch surface waves (BSWs) are presented. Desirable BSW guiding properties, such as low loss and long propagation distance, are observed. The waveguide thickness is on the order of $\lambda / 15$. At $1562 \mathrm{~nm}$ wavelength, a coupling length of $250 \mu \mathrm{m}$ is found for $3 \mu \mathrm{m}$ wide waveguides separated by $1 \mu \mathrm{m}$. The second-order mode is also investigated; we show that the fundamental mode can be excited by the second-order mode. The effect of variations in the waveguide width, gap, and refractive index are theoretically investigated by studying their impacts on the coupling length. Results are promising for mode division multiplexing, optical sensors, and optical communications. (@ 2014 Optical Society of America
\end{abstract}

OCIS codes: (220.0220) Optical design and fabrication; (230.7370) Waveguides; (240.0240) Optics at sur-

faces; (240.0310) Thin films; (240.6690) Surface waves; (220.4241) Nanostructure fabrication.

http://dx.doi.org/10.1364/JOSAB.31.002996

\section{INTRODUCTION}

Integrated optical circuits for communications applications have been an important area of research for many years. A new approach, based on using a multilayer sustaining Bloch surface waves (BSWs), is proposed as a suitable general platform for the manipulation and control of BSW propagation in the spectral range of telecom wavelengths $[\underline{1,2}]$. The platform is a one-dimensional (1D) photonic crystal, made of dielectric layers with alternating refractive indices deposited on glass. The presence of a thin additional layer on top of the photonic crystal modifies the local effective refractive index, enabling a direct manipulation of the BSW. Recently, various 2D polymer photonic components have been produced on this platform. $\mathrm{Yu}$ et al. experimentally and theoretically demonstrated that the BSW can be diffracted and focused by locally shaping the geometries of $2 \mathrm{D}$ photonic devices on the multilayer, producing elements such as lenses, prisms, and gratings [2]. Due to their dielectric nature, BSW elements provide relatively lower losses and longer propagation distances. This property is of special interest for BSW-based 2D optical circuits or sensors [3-5].

This paper will focus on a waveguide coupler consisting of parallel ultra-thin polymer waveguides (thickness $\sim \lambda / 15$ ). When the waveguides are close enough, coupling occurs between adjacent waveguides. Advantages of thin optical elements are the capability of observing propagating light in the structures using near-field measurements and the relative ease of fabrication. In the first section, we will theoretically study the coupling length as a function of different geometrical parameters. Next, the coupling process is carefully observed through measurements of near-field amplitude and phase on two BSW directional couplers. Then, a Fourier transform (FT) analysis is performed to study the mode propagation characteristics in the second waveguide coupler where multiple modes are present. This work enables understanding of the fundamental principles of evanescent coupling in BSWs and thus provides one step toward building BSW-based 2D optical devices, such as BSW switches, BSW modulators, and BSW resonators.

\section{STRUCTURE AND EXPERIMENTAL SETUP}

In this work, the multilayer structure is designed for transverse electric (TE) polarized light where the electric field is along the $y$ direction [see Fig. 1(a)], for wavelengths around $1550 \mathrm{~nm}$. It consists of six pairs of silicon nitride and silicon dioxide planar layers having refractive indices of 1.79 and 1.45 , respectively, at $\lambda=1500 \mathrm{~nm}$. The multilayer is fabricated using plasma-enhanced chemical vapor deposition. The thicknesses of the layers are 263 and $492 \mathrm{~nm}$, respectively. An extra $80 \mathrm{~nm}$ thick layer of silicon nitride is deposited on the top. The device of interest, in this case a waveguide coupler, is made of a $100 \mathrm{~nm}$ thick layer of AZ1518 photoresist (PR) $(n=1.458$, at $1500 \mathrm{~nm}$ ) and patterned by UV lithography. The excitation of the BSW is performed under total internal reflection, with a Kretschmann configuration using a BK7 glass prism in order to match the propagation constant of the BSW. The sample (glass substrate with multilayer) is placed on a prism with index matching oil. The experimental setup is shown in Fig. 1(a). The incident beam is focused and coupled and propagates initially only in the first waveguide. The light propagating in the device is then observed using a multiheterodyne scanning near-field optical microscope (MH-SNOM) developed in our lab [6,7]. The MH-SNOM allows the simultaneous 




Fig. 1. (a) Schematic diagram of the experimental setup to study the BSW waveguide directional couplers. The Kretschmann configuration with a BK7 glass prism is used in order to excite the BSW. Identical waveguides are patterned in parallel in the PR layer on top of the multilayer. The first waveguide is $120 \mu \mathrm{m}$ long to enable initial coupling of the BSW and avoid direct injection into the other waveguides of the array. (b) Top view of the waveguide coupler composed of PR ridge waveguides fabricated on the multilayer. $L$ is the waveguide length, $w$ is the waveguide width, and the gap defines the separation between adjacent waveguides.

measurement of the near-field amplitude and phase of the propagating waves in the waveguides and in the vicinity of the coupling zone $[\underline{8}, \underline{9}]$.

\section{NUMERICAL ANALYSIS}

The 3D finite-difference time-domain (FDTD) method is one of the most accurate techniques for the simulation of light propagation in nanostructures $[\underline{10,11]}$. However, when applied to large structures, extensive computation time and memory storage are needed. Therefore, an assumption valid in our case is applied in order to transform a 3D-FDTD problem into a $2 \mathrm{D}$ simulation. In the 2D-FDTD simulations, the BSW properties are first calculated by simulating the bare multilayer. The BSW mode is then injected in the plane of the top layer. The effective indices are considered for simulation instead of the refractive index of the material. In this way, we consider the coupled BSW as the incident beam, and the waveguides are considered as infinite in the $x$ direction. The BSW effective index of the bare multilayer, $n_{\mathrm{ML}}$, and the PR-coated multilayer, $n_{\mathrm{PR}}$, are deduced from their corresponding dispersion curves [2], verifying the condition $n_{\mathrm{ML}, \mathrm{PR}}=n_{g} \sin \theta$, where $n_{g}$ is the glass refractive index and $\theta$ is the incident angle. Thus, we find $n_{\mathrm{ML}}=1.1786$ and $n_{\mathrm{PR}}=1.2343$. A schematic of the simulated directional coupler is shown in Fig. 1(b). We considered 20 identical parallel waveguides in order to avoid the backcoupling of light. FDTD simulations allow the calculation of the effective index, the coupling length, and the field distribution. Numerical analyses are performed using the CST microwave studio (version 2013) commercial software based on the FDTD method. $L$, defining the length of the waveguides, is long enough in order to neglect backreflection. Experimentally, the waveguide length is on the order of centimeters, while it is $350 \mu \mathrm{m}$ in the simulations due to memory limitations. However, this length is sufficient to observe the coupling effect between adjacent waveguides.

Figure 2(a) shows the effective indices of both fundamental and second-order eigenmodes with different $\mathrm{PR}$-waveguide widths. The increase in effective index with increasing waveguide width results from the differing light distribution of the low and high refractive index media. When the width $(w)$ increases, more of the mode is found in the high index medium, and the effective index converges to the high refractive index. For a small waveguide width, while the light is confined in the waveguide, the exponential tail outside the waveguide is stronger, leading to an effective index that approaches the low refractive index. For larger $w$, the second-order mode can also be supported by the PR waveguide, and $n_{\text {eff }}$ increases with the waveguide width and approaches the fundamental mode effective index. As we can see, from $w=2 \mu \mathrm{m}$, the waveguides allow the propagation of both first- and second-order modes.

According to the coupled mode theory, studied in detail elsewhere [12], for two parallel guides the coupling length can be calculated from the following equation: $L_{c}=\pi /\left(\beta_{e}-\beta_{o}\right) . L_{c}$ corresponds to the length where the maximum power transfer occurs. $\beta_{e}$ and $\beta_{o}$ are the propagation constants of the even and odd normal modes. $L_{c}$ can be also expressed in terms of the coupling coefficient: $L_{c}=\pi / 2 k . \beta_{e}$ and $\beta_{o}$ depend on the waveguide geometries, whereas $k$ is a function of the mode overlap in the waveguide coupler. It strongly depends on the separation between adjacent waveguides. In the case of three identical waveguides, the coupling length depends on the waveguide used for injection. When an outer waveguide is fed, the coupling length for an input beam on waveguide number 1 to be completely coupled to waveguide number 3 is $L_{c}=2 \pi /\left(\beta_{e}-\beta_{o}\right)$. It is two times longer than the coupling
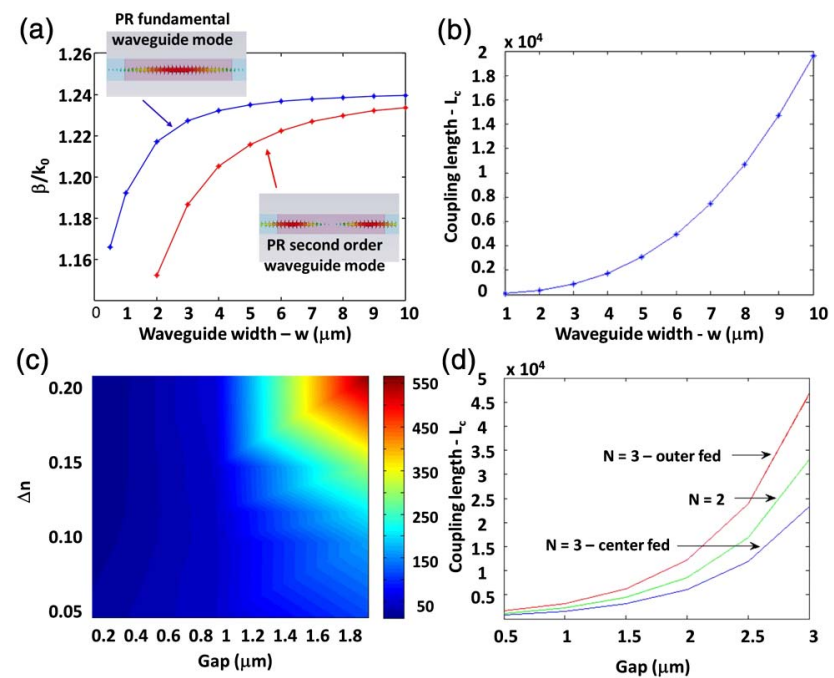

Fig. 2. (a) Effective index of the dielectric waveguide modes versus the width of the waveguide. The field distributions of the waveguide modes are shown in the insets. (b) With $\Delta n=0.055$, gap $=1 \mu \mathrm{m}$, coupling length as a function of waveguide width, $w$. (c) $L_{c}$ as a function of the gap spacing and the waveguide refractive index, keeping $w=3 \mu \mathrm{m}$. (d) With $\Delta n=0.055, L_{c}$ for two and three waveguide couplers (outer and center fed). The waveguide width is fixed at $3 \mu \mathrm{m}$. 
length with the center waveguide feed [13]. $\beta_{e}$ and $\beta_{o}$ correspond to the symmetric (even) and antisymmetric (odd) modes, respectively.

A variety of examples that address the effect of varying parameters including the gap, waveguide width, number of parallel waveguides, and refractive index contrast are presented in this section. The refractive index contrast is created when adding an additional layer on the platform. It corresponds to the difference between the effective index of the BSW mode when excited with the bare multilayer (platform) and the effective index for the BSW mode when an additional layer is added. First, the refractive index contrast is fixed at 0.055 , and the waveguide width varies from 1 to $10 \mu \mathrm{m}$. The coupling length is calculated and plotted in Fig. 2(b). Increasing the width, $w$, results in an increase in the waveguide propagation constant of both symmetric and asymmetric modes. For high $w$, the propagation constant of the second-order mode remains closer to the fundamental one, leading to a strong increase of the coupling length. The coupling length depends on other physical parameters: the gap spacing, refractive index contrast, and total number of waveguides. Figure 2(c) shows the plots of $L_{c}$ as a function of gap and $\Delta n$, and Fig. $\overline{2(\mathrm{~d})}$ shows plots of $L_{c}$ as a function of gap and $N$, where $N$ is the number of waveguides considered. One can see that $L_{c}$ is strongly dependent on these parameters. First, in Fig. 2(c), $L_{c}$ increases with both the gap spacing and $\Delta n$. This is expected because increasing the separation distance or confinement of the mode results in a reduction of the coupling efficiency and thus an increase of the corresponding coupling length. Figure 2(d) shows that when varying the guide separation, the threewaveguide coupler with center feed has lower $L_{c}$, whereas the three-waveguide coupler with outer-guide feed needs a longer length for a total transfer of energy.

In summary, one can scale the design of the waveguide components with a given index contrast to obtain the desired coupling length.

\section{EXPERIMENTS AND DISCUSSION}

First, a near-field measurement is performed on a BSW directional coupler with $3 \mu \mathrm{m}$ wide waveguides separated by a gap of $1 \mu \mathrm{m}$, as shown in Fig. 3(b). The near-field scan covers the first (left-most) 6 of the 20 identical waveguides on the sample. Experimentally, the incident beam having a fixed wavelength of $\lambda=1562 \mathrm{~nm}$ is injected into the directional coupler with an incidence angle of $54^{\circ}$. Both the simulated electric field distribution and the experimental near-field measurements are compared in Figs. 3(a) and 3(b), respectively. As can be seen, after launching light into the left-most waveguide, a complete power transfer to the adjacent guides occurs after a propagation distance of $\sim 250 \mu \mathrm{m}$. From both the measured and simulated field distributions, wavy patterns are observed, resulting from the interference between the coupled modes [14]. The experimental results in Fig. 3(c) show the power transfer from the first to the adjacent waveguide. Figure $3(\mathrm{~d})$ presents the intensity profile at $z=140 \mu \mathrm{m}$ compared to the device topography at the same location. It illustrates that there is high field amplitude in the second waveguide from the left and a weak field in the surrounding region.

Second, for comparison purposes, near-field measurements are performed on another directional coupler with $w=2 \mu \mathrm{m}$, gap $=2 \mu \mathrm{m}$. The simulated and measured near-field amplitudes
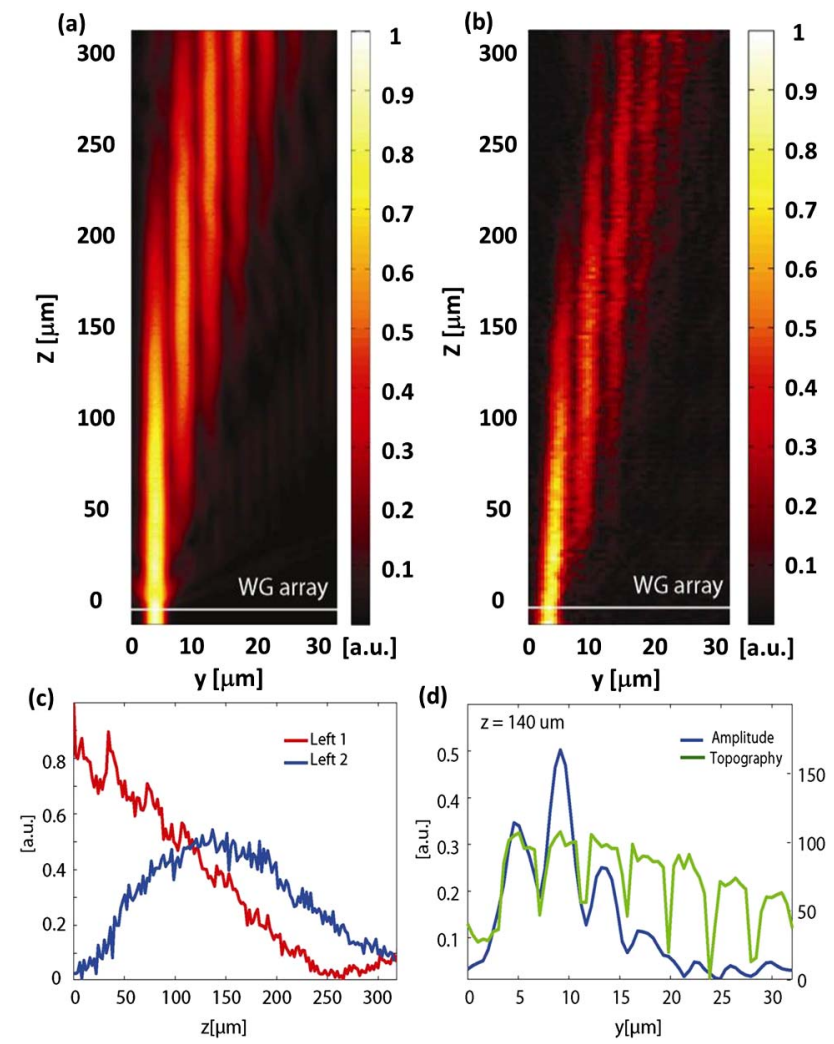

Fig. 3. Single mode coupling on a $w=3 \mu \mathrm{m}$, gap $=1 \mu \mathrm{m}$ waveguide coupler which contains 20 identical waveguides. (a) Simulated (FDTD method) electric field amplitude in comparison with (b) experimental near-field measurement results. (c) Field amplitude taken from the first and second waveguide (from left) along $z$, where the observed coupling length indicates the energy exchange. (d) Amplitude profile at $z=140 \mu \mathrm{m}$ in (b) compared to the topography measurement at the same location. The position of the peak field amplitude clearly shows that the light is coupled into the second waveguide from the left.

are shown in Figs. $\underline{4(\mathrm{a})}$ and $\underline{4(\mathrm{~b})}$, respectively. The first (antisymmetric) mode is excited and propagates along the left-most waveguide, which is $28 \mu \mathrm{m}$ longer than the rest of the waveguides in order to enable an initial coupling of the BSW and to avoid direct injection into the other waveguides of the array. The laser wavelength is maintained at $1562 \mathrm{~nm}$.

The cross-section profiles of the measured near-field amplitude and topography at different $z$ positions along the leftmost seven waveguides are shown in Figs. $\underline{4(\mathrm{c})}$ and $\underline{4(\mathrm{~d})}$. The measured phase maps of the regions indicated by the gray squares are shown in Figs. $\underline{4(\mathrm{e})}$ and $\underline{4(\mathrm{f})}$. In Fig. $\underline{4(\mathrm{c})}$, we see a cross-section profile in the region shortly after entering the waveguide array region $(z=14 \mu \mathrm{m})$, where the symmetric mode in the left-most guide is strongest; light is confined at the edges and lies in between the waveguides. The $\pi$ shift in the phase map shown in Fig. 4(e) indicates clearly the $m=$ 1 mode. In Fig. $\underline{4(\mathrm{~d})}(z=254 \mu \mathrm{m})$, we observe that energy has been coupled into the adjacent guides as the maximum energy is in the second waveguide from the left. Figure $4(\mathrm{f})$ shows that the fundamental mode (asymmetric) is excited, and light is well confined in the waveguides.

An analysis of the mode propagation in the second waveguide coupler is performed using the FT. The experimental data is based on the undersampled near-field amplitude and phase measurements of the propagating wave as shown in 

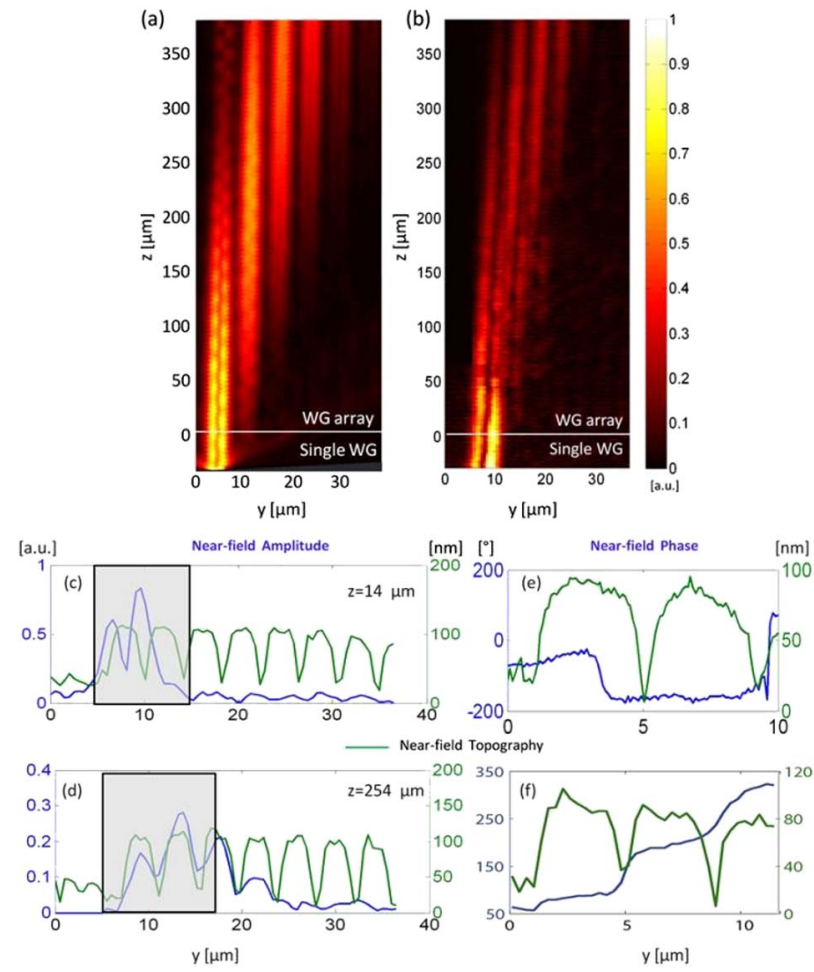

Fig. 4. (a) Simulated and (b) measured near-field amplitude on the $w=2 \mu \mathrm{m}$, gap $=2 \mu \mathrm{m}$ directional coupler. The injection zone is on the left-most single waveguide (WG). The single waveguide joins the waveguide array at $z=0 \mu \mathrm{m}$; cross-section profiles of the measured near-field amplitude and topography for (c) $z=14$ and (d) $z=254 \mu \mathrm{m}$; (e), (f) Measured phase maps of the regions indicated in gray in (c) and (d), respectively; the $\pi$ phase shift illustrates the second mode in (e).

Fig. 4(b). Figure $\underline{5(\mathrm{a})}$ shows the FT along the propagation (z) direction of the area indicated by the dashed lines in the inset. The region covers the first (left-most) waveguide [shown in Fig. 4(b)] from $z=12$ to $172 \mu \mathrm{m}$ with 81 sample points (sample spacing of $2 \mu \mathrm{m}$ ). Similarly, in Fig. 5(b), we show the FT for the second waveguide but in the region from $z=272$ to $382 \mu \mathrm{m}$ with 56 sample points (sample spacing also $2 \mu \mathrm{m}$ ), again indicated by the dashed area in the inset. Given that the sample spacing is $2 \mu \mathrm{m}$, the frequency span shown in Figs. 5(a) and 5(b) ranges from 0 to $0.5 \mu \mathrm{m}^{-1}$. These data are then averaged in the transverse $(y)$ direction, producing the spectra shown in Figs. 5(c) and 5(d). In Fig. 5(c), we see four significant peaks in the spectrum, located at values of $0.216,0.235,0.259$, and $0.272 \mu \mathrm{m}^{-1}$. In Fig. 5(d), we have one significant peak at $0.268 \mu \mathrm{m}^{-1}$. For the given number of measurement points, the resolution of each spectrum is approximately 0.00617 and $0.00893 \mu \mathrm{m}^{-1}$, respectively. Within the resolution of the measurement data, the peak in Fig. 5(d) and the right-most peak in Fig. $\underline{5(\mathrm{c})}$ can be considered to be at the same frequency.

Theoretically, the propagation constants are calculated using the CST microwave studio 2D mode solver. As discussed in Section 1, the computed values depend strongly on the geometrical parameters of the structure. Simulations show that a $2 \mu \mathrm{m}$ wide waveguide with a $2 \mu \mathrm{m}$ gap distance has a propagation constant of $0.7771 \mu \mathrm{m}^{-1}$ for the fundamental mode and $0.7355 \mu \mathrm{m}^{-1}$ for the second mode. Since the sampling frequency used in the experimental near-field measurements

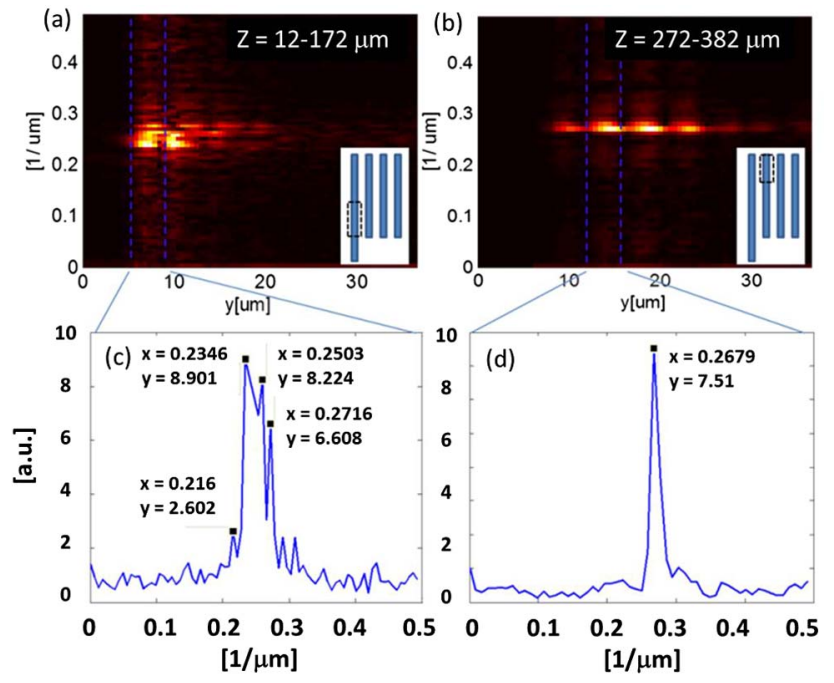

Fig. 5. FT performed on the complex near-field measurement data corresponding to the $y-z$ area (a) in the left-most guide for $z=12-172 \mu \mathrm{m}$, and (b) in the second guide from the left for $z=$ 272-382 $\mu \mathrm{m}$ [see Fig. 4(b)]. The FT is performed along $z$ for each $y$

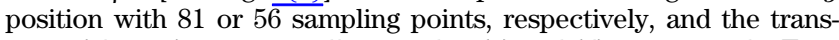
verse (along $y$ ) average is illustrated in (c) and (d), respectively. Four main peaks of $0.216,0.235,0.259$, and $0.272 \mu \mathrm{m}^{-1}$ are found within the dashed area around the left-most waveguide in (a). Similarly, one peak of $0.268 \mu \mathrm{m}^{-1}$ is found within the dashed area around the second leftmost waveguide in (b).

is well below the Nyquist frequency for the propagating waves, we expect to have aliasing effects in the spectrum. The frequency span of the spectra is $0.5 \mu \mathrm{m}^{-1}$, given the constant $2 \mu \mathrm{m}$ scan spacing, meaning that the frequency peaks in the results can be expected to be found with an integer multiple of this frequency span value added or subtracted. The peaks described above at $0.272 \mu \mathrm{m}^{-1}$ in Fig. $\underline{5(\mathrm{c})}$ and at $0.268 \mu \mathrm{m}^{-1}$ in Fig. $5(\mathrm{~d})$ correspond to the predicted values of the propagation constant for the fundamental mode. The peak in Fig. 5(c) at frequency $0.235 \mu \mathrm{m}^{-1}$ shifted by one frequency span also corresponds to the predicted frequency of the second mode. The remaining two peaks in Fig. 5(c) are the approximate complements of the two frequency values discussed above, modulo the frequency span of $0.5 \mu \mathrm{m}^{-1} \quad\left(0.272+0.216=0.488 \mu \mathrm{m}^{-1}\right.$ and $0.235+0.259=$ $\left.0.494 \mu \mathrm{m}^{-1}\right)$. These peaks correspond to the backward propagating fundamental and second modes of the input waveguide. Considering the relative amplitudes of the four peaks in Fig. 5(c), this suggests that the forward-propagating second mode is the dominant signal, followed by the backward-propagating second mode, the forward-propagating fundamental mode, and lastly the backward-propagating fundamental mode (with a significantly lower amplitude). In Fig. 5(d), considering the second waveguide far from the input coupler region, we see that only the forward-propagating fundamental mode is seen.

\section{CONCLUSIONS}

In conclusion, a waveguide directional coupler fabricated on an ultra-thin $(\sim \lambda / 15)$ BSW-sustaining multilayer has been studied experimentally and theoretically. First, the impacts of the refractive index contrast, gap separation, and waveguide width on the coupling length are investigated in simulation. Then the performance of example devices is verified 
experimentally. Two waveguide couplers with different geometries are studied. The coupling and energy transfer process is carefully observed through measurements of the near-field amplitude and phase. The coupling length is calculated and compared with the measured field distribution. Comparing the two measured waveguide couplers, the geometry of the second device has a reduced guide width and an increased gap. As expected from the theoretical prediction, the observed coupling length increases in the second case. This is because after the second mode vanishes, only the fundamental mode is left, and the effect of the waveguide gap is greater than that of the guide width. This study contributes to understanding and designing the evanescent coupling function in BSW devices such as BSW switches, BSW modulators, and BSW resonators in BSW-based optical communication, all optical integrated optical systems, and optical sensors.

\section{ACKNOWLEDGMENTS}

The authors acknowledge support from the Swiss National Science Foundation in the frame of the SNOM and SWAP projects.

\section{REFERENCES}

1. P. Yeh, A. Yariv, and C.-S. Hong, "Electromagnetic propagation in periodic stratified media. I. General theory," J. Opt. Soc. Am. 67, 423-438 (1977)

2. L. Yu, E. Barakat, V. Paeder, L. Hvozdara, J. D. Francesco, and H. P. Herzig, "Manipulate Bloch surface wave with 2D dielectric elements: platform concept based flat lens," Light Sci. Appl. 3, e124 (2014).

3. F. Giorgis, E. Descrovi, C. Summonte, L. Dominici, and F. Michelotti, "Experimental determination of the sensitivity of Bloch surface waves based sensors," Opt. Express 18, 8087-8093 (2010).
4. V. Paeder, V. Musi, L. Hvozdara, S. Herminjard, and H. P. Herzig, "Detection of protein aggregation with a Bloch surface wave based sensor," Sens. Actuators B 157, 260-264 (2011).

5. S. Santi, V. Musi, E. Descrovi, J. Di Francesco, L. Hvozdara, P. van der Wal, H. A. Lashuel, A. Pastore, R. Neier, and H. P. Herzig, "Real-time amyloid aggregation monitoring with a photonic crystal based approach," Chem. Phys. Chem. 14, 3476-3482 (2013).

6. E. Descrovi, T. Sfez, L. Dominici, W. Nakagawa, F. Michelotti, and H. P. Herzig, "Near-field imaging of Bloch surface waves on silicon nitride one-dimensional photonic crystals," Opt. Express 16, 5453-5464 (2008).

7. T. Sfez, "Investigation of surface electromagnetic waves with multi-heterodyne scanning near-field optical microscopy," Master's Thesis (École Polytechnique Fédérale de Lausanne, 2010).

8. L. Yu, T. Sfez, V. Paeder, P. Stenberg, W. Nakagawa, M. Kuittinen, and H. P. Herzig, "Concurrent polarization retrieval in multi-heterodyne scanning near-field optical microscopy: validation on silicon form-birefringent grating," Opt. Express 20, 23088-23099 (2012).

9. T. Sfez, E. Descrovi, L. Yu, D. Brunazzo, M. Quaglio, L. Dominici, W. Nakagawa, F. Michelotti, F. Giorgis, O. J. F. Martin, and H. P. Herzig, "Bloch surface waves in ultrathin waveguides: near-field investigation of mode polarization and propagation," J. Opt. Soc Am. B 27, 1617-1625 (2010).

10. K. Yee, "Numerical solution of initial boundary value problems involving Maxwell's equations in isotropic media," IEEE Trans. Antennas Propag. 14, 302-307 (1966).

11. A. Taflove and M. E. Brodwin, "Numerical solution of steadystate electromagnetic scattering problems using the timedependent Maxwell's equations," IEEE Trans. Microwave Theor. Tech. 23, 623-630, 1975.

12. A. Yariv, Quantum Electronics, 3rd ed. (Wiley, 1989), p. 27.

13. Y.-M. Kim, S.-P. Han, and C.-M. Kim, "Crosstalks of twowaveguide and three-waveguide directional couplers," Opt. Quantum Electron. 32, 1257-1268 (2000).

14. M. Abashin, P. Tortora, I. Märki, U. Levy, W. Nakagawa, L. Vaccaro, H. P. Herzig, and Y. Fainman, "Near-field characterization of propagating optical modes in photonic crystal waveguides," Opt. Express 14, 1643-1657 (2006). 